Pacific Journal of Mathematics

A SOLUTION FOR SCATTERED ORDER TYPES OF A 


\title{
A SOLUTION FOR SCATTERED ORDER TYPES OF A PROBLEM OF HAGENDORF
}

\author{
JEAN A. LARSON
}

J. Hagendorf asked if every order type $\phi$ having the following two properties of additively indecomposable ordinals was the order type of an ordinal. Call $\phi$ Hagendorf if (i) it is strictly indecomposable to the right, i.e., if $\phi=\phi+\theta$, then $\phi$ can be embedded in $\theta$ but not in $\phi$, and (ii) every strictly smaller type can be embedded in an initial segment of $\phi$, i.e., if $\chi$ can be embedded in $\phi$ but not vice versa, then $\phi=\phi+\theta$ where $\theta \neq 0$ and $\chi$ can be embedded in $\phi$. Recall that scattered order types are those which do not embed the order type of the rationals.

The paper provides a partial answer to Hagendorf's question: Every scattered Hagendorf type is the order type of an indecomposable ordinal.

Other subclasses of order types for which this question seems particularly interesting are sub-types of the order type of the real numbers, and the class of countable unions of scattered types.

If $\phi$ and $\psi$ are order types of linearly ordered sets, then say $\phi$ is embeddable in $\psi$, and write $\phi \leqq \psi$ if for any representatives $L$ and $M$ of $\phi$ and $\psi$ respectively, there is an embedding (one-to-one order-preserving function) of $L$ into $M$. Write $\phi<\psi$ if $\phi \leqq \psi$ and $\psi \not \phi$.

Above strictly indecomposable to the right is defined. The definition of strictly indecomposable to the left is made analogously. If $\phi$ is strictly indecomposable to the right, then call $\psi$ a proper segment of $\phi$, if $\phi$ is strictly indecomposable to the right and $\phi=$ $\psi+\theta$ where $\theta \neq 0$ or if $\phi$ is strictly indecomposable to the left and $\dot{\phi}=\theta+\psi$ where $\theta \neq 0$. If $\phi$ is strictly indecomposable to the right or left, then write $\psi \ll \theta$ if $\psi$ is embeddable in a proper segment of $\theta$. With this notation, $\phi$ is Hagendorf, if $\phi$ is strictly indecomposable to the right, and for all $\psi<\phi$, it is true that $\psi \ll \phi$. In this case, write $\phi \in J H$.

Identify an ordinal with the set of its predecessors and with the order type of that set under $\varepsilon$. Then every additively indecomposable ordinal $\alpha$ is strictly indecomposable to the right, and has the further property that if $L \leqq \alpha$ and $\alpha$ cannot be embedded in $L$, then $L$ can be embedded in a proper initial segment of $\alpha$.

At the time he told me of Hagendorf's problem, F. Galvin sketched for me the proof that all countable Hagendorf types are 
ordinals as follows. Suppose by way of contradiction that $\phi$ is a countable Hagendorf type that is not an ordinal. Then $\phi$ embeds every countable ordinal. (The proof is by induction. If $\alpha \leqq \phi$, then $\alpha<\phi$ so $\alpha \ll \phi$ and $\alpha+1 \leqq \phi$. Similarly, if $\alpha_{n} \leqq \phi$ for all $n<\omega$, then $\left.\alpha=\Sigma \alpha_{n} \leqq \phi.\right)$ However, if $\phi$ embeds all countable ordinals, then either $\phi$ embeds $\eta$, the order type of the rationals, or $\phi$ embeds $\omega_{1}$ or $\omega_{1}^{*}$. The latter is clearly impossible because $\phi$ is countable. And if $\eta \leqq \phi$, then $\phi$ is not strictly indecomposable to the right, because $\eta$ embeds every countable order type. But $\phi$ is strictly indecomposable to the right, since $\phi$ is Hagendorf. This contradiction yields the claim that there is no countable Hagendorf type which is not the order type of an ordinal.

The proof that there is no scattered Hagendorf type that is not the order type of an ordinal is analogous in that it consists of showing that such a type would embed all converse ordinals.

The embeding relation gives rise to an equivalence relation on order types: $\phi \equiv \psi$ if and only if $\phi \leqq \psi$ and $\psi \leqq \phi$.

Lemma. If $\phi \in J H$ and $\psi \equiv \phi$, then $\psi \in J H$.

The proof is left as an exercise for the reader.

The set theoretic terminology of this paper is standard. As the Axiom of Choice is assumed throughout, cardinals and initial ordinals of that cardinality are identified. The cofinality of an ordinal $\alpha$, cf $\alpha$, is the smallest cardinal $\nu$ so that there is a set $X \cong \alpha$ with $|X|=\nu$ which is unbounded in $\alpha$. Write $0 n$ for the class of ordinals. If for each $\beta<\mu$, the linearly ordered set $\left(A_{\beta}, \leqq\right)$ is a representative of $\phi_{\beta}$, then $\sum_{\beta<\mu}^{*} \phi_{\beta}$ is the order type of the disjoint union $\bigcup_{\beta<\mu} A_{\beta}$ ordered by the relation $x \leqq y$ if and only if $\beta>\gamma$, or $\beta=\gamma$ and $x \leqq y$ in $A_{\beta}$. This order type is the converse well-ordered sum of the sequence $\phi_{\beta}$.

If $\phi$ is an order type with representative $(A, \leqq)$, then $\phi^{*}$ is the order type determined by $\left(A, \geqq^{*}\right)=(A, \geqq)$, i.e., $a \geqq^{*} b$ if and only if $b \leqq a$. An order type $\phi$ is additively indecomposable if whenever $\phi=\psi+\theta$, either $\phi \leqq \psi$ or $\phi \leqq \theta$.

Denote by $\mathscr{S}$ the class of all scattered order types, that is, those which do not embed $\eta$, the order type of the rationals. Hausdorf introduced a hierarchy of the scattered order types.

THEOREM (F. Hausdorf [2]).

$$
\mathscr{S}=\bigcup_{\alpha \in 0 n} \mathscr{S}_{\alpha}
$$


where

$\mathscr{S}_{0}=$ the set of order types $\{0,1\}$, and for $\beta>0$,

$\mathscr{S}_{\beta}=\{\phi: \phi$ is a well-ordered or converse well-ordered sum of members of $\mathbf{U}_{r<\beta} \mathscr{S}_{\gamma}$.

THEOREM 1. Every scattered Hagendorf type is the order type of an ordinal.

Proof. The next few paragraphs outline the proof.

Assume by way of contradiction that $\phi \in \mathscr{S} \cap J H$ and $\phi \notin 0 n$. Let $\gamma$ be the limit of all ordinals which are embeddable in $\phi$. Prove in Lemma 2, that $\gamma$ is a limit ordinal and $\gamma \not \$ \phi$. Write $\gamma=\sum_{\beta<\lambda} \gamma_{\beta}$ where $\lambda=\operatorname{cf} \gamma$, and let $\theta=\sum_{\beta<\lambda}^{*} \gamma_{\beta}$. Prove that $\theta \leqq \phi$ by proving an embedding lemma (Lemma 3 ) which says that for certain wellordered sequences, called regular segmented sequences, if all the members of the sequence are embeddable in $\phi$, then so is either the well-ordered sum or the converse well-ordered sum of the sequence.

Next define $\mathscr{D}$ as the closure of $\{0,1\}$ under multiplication by indecomposable ordinals and converse ordinals, and under well-ordered sums and converse well-ordered sums of regular segmented sequences. In Lemma 8, show for $\delta \in \mathscr{D}$ with $|\delta|<\lambda$ and for certain $\pi$, that order types of the form $\theta \cdot \pi \cdot \delta \notin J H$.

Using that result and the embedding lemma, show that for every $\alpha \in 0 n,\left(\lambda^{\alpha}\right)^{*} \leqq \theta \cdot\left(\lambda^{\alpha}\right)^{*} \leqq \phi$, the contradiction that proves the theorem.

Now the details of the proof are given starting from the assumption that $\phi \in \mathscr{S} \cap J H$ and $\phi \notin 0 n$.

LEMMA 2. Let $\gamma$ be the limit of all ordinals embeddable in $\phi$. Then $\gamma \not \phi, \gamma$ is a limit, and $\lambda=\operatorname{cf} \gamma>\omega$.

Proof. If $\beta$ is an ordinal, then $\phi \npreceq \beta$ since $\phi$ is not an ordinal, and every order type embeddable in $\beta$ is that of an ordinal. If $\beta \leqq \phi$, then $\beta<\phi$, so $\beta \ll \phi$, so $\beta+1 \leqq \phi$. Thus there is no largest ordinal embeddable in $\phi$, and the limit $\gamma$ of all ordinals embeddable in $\phi$ is a limit ordinal. (Notice that the ordinals embeddable in $\phi$ are bounded above by $|\phi|^{+}=|L|^{+}$where $L$ is a representative of $\left.\phi.\right)$ Further ef $\gamma=\lambda>\omega$, since if cf $\gamma=\omega$, then $\gamma \leqq \phi$.

Definition. A sequence $\left\langle\psi_{\alpha}: \alpha<\mu\right\rangle$ of order types is a regular segmented sequence if and only if $\mu$ is a regular cardinal, each $\psi_{\alpha}$ is strictly indecomposable to the right or to the left, and for all $\alpha, \beta$ with $\alpha<\beta<\mu, \psi_{\alpha} \ll \psi_{\beta}$. 
Lemma 3 (Embedding Lemma). If $\chi \in \mathscr{S}$ and $\left\langle\psi_{\alpha}: \alpha<\mu\right\rangle$ is a regular segmented sequence and for all $\alpha<\mu, \psi_{\alpha} \leqq \chi$, then either $\sum_{\alpha<\mu} \psi_{\alpha} \leqq \chi$ or $\sum_{\alpha<\mu}^{*} \psi_{\alpha} \leqq \chi$.

Proof. Fix some regular segmented sequence $\left\langle\psi_{\alpha}: \alpha\langle\mu\rangle\right.$. Let $\mathscr{T} \cong \mathscr{S}$ be the collection of scattered order types for which the lemma is true.

Since 0 and 1 are vacuously in $\mathscr{T}$, it suffices to show that $\mathscr{T}$ is closed under well-ordered and converse well-ordered sums. The proof proceeds by induction on the ordinal used in indexing the sum.

Any sum with zero or one term from $\mathscr{T}$ is in $\mathscr{T}$. Suppose $\tau>1$ is an ordinal and every well-ordered sum and converse wellordered sum of fewer than $\tau$ terms from $\mathscr{T}$ is in $\mathscr{T}$. Assume $\chi=\sum_{\xi<\tau} \chi_{\xi}$ is a well-ordered sum of $\tau$ terms from $\mathscr{T}$. The case of a converse well-ordered sum is handled analogously. If for some $\alpha<\mu, \psi_{\alpha} \leqq \$, then $\chi$ is in $\mathscr{T}$. So assume for all $\alpha<\mu, \psi_{\alpha} \leqq \chi$.

Suppose $\tau=t+1$. Since each $\psi_{\alpha}$ is additively indecomposable and embeddable in all $\psi_{\beta}$ with $\beta>\alpha$, either for all $\alpha<\mu, \psi_{\alpha} \leqq$ $\sum_{\xi<t} \chi_{\xi}$ or for all $\alpha<\mu, \psi_{\alpha} \leqq \chi_{t}$. In either case, we are done by the induction hypothesis.

So suppose $\tau$ is a limit. Notice that for all $\alpha<\mu$, since $\psi_{\alpha} \ll$ $\psi_{\alpha+1} \leqq \chi$, there is a smallest ordinal $s(\alpha)<\tau$ so that $\psi_{\alpha} \leqq \sum_{\xi<s(\alpha)} \chi_{\xi}$. The function $s: \mu \rightarrow \tau$ is nondecreasing. If it is bounded above by some $\nu<\tau$, then the induction hypothesis insures $\chi \in \mathscr{T}$.

If $s$ is not bounded above, then $\mathrm{cf} \tau=\mu$, and for all $t<\tau$, for all $\alpha<\mu, \psi_{\alpha}$ is embeddable in an initial segment of $\sum_{t \leqq \xi<\tau} \chi_{\xi}$, since for some $\beta>\alpha, \psi_{\beta} \not \sum_{\varepsilon<t} \chi_{\xi}$ and $\psi_{\beta}$ is additively indecomposable. In this case define a function $t: \mu \rightarrow \tau$ by recursion so that for all $\alpha<\mu, \psi_{\alpha} \leqq \sum_{t(\alpha) \leqq \xi<t(\alpha+1)} \chi_{\xi}$. Use this function to see $\chi \in \mathscr{T}$.

COROLLARY 4. Let $\lambda=\operatorname{cf} \gamma$ and let $\left\langle\gamma_{\alpha}: \alpha<\lambda\right\rangle$ be a strictly increasing sequence of ordinals with limit $\gamma$. Then $\theta=\sum_{\alpha<\lambda}^{*} \gamma_{\alpha} \leqq \phi$.

Recall that $\mathscr{D}$ is the closure of $\{0,1\}$ under multiplication by additively indecomposable ordinals and converse ordinals and under well-ordered sums and converse well-ordered sums of regular segmented sequences. The next few lemmas summarize the properties of $\mathscr{D}$.

Lemma 5. For all $d \in \mathscr{D}, d$ is additively indecomposable.

Proof. First notice that 0 and 1 are additively indecomposable. Next check that if $a \in \mathscr{S}, b>1$, and $b$ is indecomposable, so is $a \cdot b$, particularly in the case $b$ an ordinal or converse ordinal. Finally 
check that the well-ordered sum and the converse well-ordered sum of a regular segmented sequence are always additively indecomposable.

LeMma 6. If $a, b \in \mathscr{S}, b$ is additively indecomposable, and $a \nless b$, then for all $d \in \mathscr{D}, a \cdot d \nless b \cdot d$.

Proof. Let $a, b$ be given. Let $\mathscr{E}=\{d \in \mathscr{D}: a \cdot b \nless b \cdot d\}$. The lemma is clearly true for $d=0$ and $d=1$, so $\{0,1\} \subseteq \mathscr{E}$.

Claim $1 . \mathscr{E}$ is closed under multiplication by additively indecomposable ordinals and converse ordinals.

The proof of Claim 1 proceeds by induction on the additively indecomposable ordinals. Assume $d \in \mathscr{E}$. Then $d \cdot 1=d=d \cdot 1^{*} \in \mathscr{E}$. Let $\beta$ be a given additively indecomposable ordinal and suppose for all $\rho<\beta$ with $\rho$ additively indecomposable, $a \cdot d \cdot \rho \nless b \cdot d \cdot \rho$ and $a \cdot d \cdot \rho^{*} \nless b \cdot d \cdot \rho^{*}$. Suppose $a \cdot d \cdot \beta \ll b \cdot d \cdot \beta$. Then for some $\eta<\beta$, $a \cdot d \cdot \beta \leqq b \cdot d \cdot \eta$. The ordinal $\eta$ can be decomposed as a finite sum of additively indecomposable ordinals. This decomposition induces a decomposition of $b \cdot d \cdot \eta$ into the sum of finitely many additively indecomposable ordinals. Since $a \cdot d \cdot \beta$ is additively indecomposable, for some member $b \cdot d \cdot \rho$ of the sum which makes up $b \cdot d \cdot \eta$, the inequality $a \cdot d \cdot \beta \leqq b \cdot d \cdot \rho$ holds. Thus the contradiction $a \cdot d \cdot \rho \ll$ $b \cdot d \cdot \rho$ is reached. A similar argument shows that the assumption $\alpha \cdot d \cdot \beta^{*} \ll b \cdot d \cdot \beta^{*}$ leads to a contradiction.

Claim 2. $\mathscr{E}=\{d \in \mathscr{D}: a \cdot d \nless b \cdot d\}$ is closed under well-ordered sums and converse well-ordered sums of regular segmented sequences.

Let $\left\langle d_{\eta}: \eta\langle\nu\rangle\right.$ be a regular segmented sequence, and suppose $d_{\eta} \in \mathscr{E}$ for $\eta<\nu$. Let $d=\sum_{\eta<\nu} d_{\eta}$. Assume by way of contradiction, that $a \cdot d \ll b \cdot d$. That is, for some $\rho<\nu, a \cdot d \leqq b \cdot \sum_{\eta<\rho} d_{\eta}$.

If $\nu=\omega$, then for some $n<\omega, a \cdot d \leqq b \cdot d_{n}$, so $a \cdot d_{n} \ll b \cdot d_{n}$, a contradiction to $d_{n} \in \mathscr{E}$. So assume $\nu>\omega$. Since $d \in \mathscr{D}, d$ and $a \cdot d$ are additively indecomposable. Thus without loss of generality, $\rho$ may be assumed to be additively indecomposable. Note that $a \cdot d_{\rho} \cdot \rho \ll$ $a \cdot d \leqq b \cdot \sum_{\eta<\rho} d_{\eta} \leqq b \cdot d_{\rho} \cdot \rho$, since $\left\langle d_{\eta}: \eta\langle\nu\rangle\right.$ is a regular segmented sequence. Thus $a \cdot d_{\rho} \cdot \rho \ll b \cdot d_{\rho} \cdot \rho$, a contradiction to the facts that $d_{\rho} \in \mathscr{E}$ and $\mathscr{E}$ is closed under multiplication by additively indecomposable ordinals. Thus $\mathscr{E}$ is closed under well-ordered sums of regular segmented sequences. The proof for converse well-ordered sums is analogous.

Claims 1 and 2 show that $\mathscr{E}$ has the closure properties of $\mathscr{D}$, 
LEMMA 7. $\lambda^{*} \nless \theta$.

Recall $\theta=\sum_{\alpha<\lambda}^{*} \gamma_{\alpha}$ where $\gamma=\sum_{\alpha<\lambda} \gamma_{\alpha}$ and $\lambda$ is the cofinality of $\gamma$. The proof is a straightforward induction on ordinals less than $\lambda$.

LEMMA 8. If $\pi$ is an additively indecomposable converse ordinal or a well-ordered sum of a regular segmented sequence of fewer than $\lambda$ converse ordinals, then for all $d \in \mathscr{D}$ with $|d|<\lambda, \theta \cdot \pi \cdot d \notin J H$.

Proof. Let $\psi=\theta \cdot \pi \cdot d$, and let $\chi=\lambda^{*} \cdot \pi \cdot d$. If $\pi$ is a wellordered sum of a regular segmented sequence of fewer than $\lambda$ converse ordinals, let $\left\langle\pi_{\alpha}: \alpha\langle\rho\rangle\right.$ be such a sequence.

Clearly $\chi \leqq \psi$. The previous two lemmas imply that $\chi \nless \psi$. To show that $\psi \notin J H$, it suffices to show that $\chi<\psi$, i.e., $\psi \geqq \chi$.

Assume by way of contradiction that $\psi \leqq \chi$. Then $\theta \leqq \chi$, so every ordinal $\xi$ embeddable in $\gamma$ is embeddable in $\chi$. However every ordinal which can be embedded in $\chi$ can be embedded in $\omega \cdot d$ if $\pi$ is a converse ordinal, or in $\omega \cdot \rho \cdot d$ if $\pi=\sum_{\alpha<\rho} \pi_{\alpha}$. Now the embedding lemma either implies that $(\gamma \leqq \omega \cdot d$ or $\theta \leqq \omega \cdot d)$ or implies that $(\gamma \leqq \omega \cdot \rho \cdot d$ or $\theta \leqq \omega \cdot \rho \cdot d)$. Thus either $\lambda \leqq|\gamma|=|\theta| \leqq|\omega \cdot d|<\lambda$ or $\lambda \leqq|\gamma|=|\theta| \leqq|\omega \cdot \rho \cdot d|<\lambda$. In both cases, the contradiction $\lambda<\lambda$ yields the desired conclusion $\psi \not \equiv \chi$.

Lemma 9. Let $\pi$ be an additively indecomposable ordinal or the well-ordered sum of a regular segmented sequence of fewer than $\lambda$ converse ordinals. If $\theta \cdot \pi \leqq \phi$, then $\theta \cdot \pi \cdot \lambda^{*} \leqq \phi$.

Proof. Define by recursion, a sequence $\left\langle d_{\alpha}: \alpha\langle\lambda\rangle\right.$ of members of $\mathscr{D}$ each of cardinality $<\lambda$. Set $d_{0}=\omega$. If $d_{\alpha}$ has been defined, set $d_{\alpha+1}=d_{\alpha} \cdot \omega$. If $\alpha$ is a limit ordinal and for $\beta<\alpha, d_{\beta}$ has been defined, select a cofinal set $I \subseteq \alpha$ of ordinals so that $I$ has the order type of a regular cardinal, and set $d_{\alpha}=\sum_{\beta \in I} d_{\beta}$ if that sum is embeddable in $\phi$, and set $d_{\alpha}=\sum_{\beta \in I}^{*} d_{\beta}$ otherwise.

Prove by induction that $\left\langle d_{\alpha}: \alpha\langle\lambda\rangle\right.$ is a regular segmented sequence. Also by induction prove that for all $\alpha<\lambda, \theta \cdot \pi \cdot d_{\alpha} \leqq \phi$, using the embedding lemma at limit stages, and another induction argument at successor stages. For if $\theta \cdot \pi \cdot d_{\beta} \leqq \phi$, since by Lemma $8, \theta \cdot \pi \cdot d_{\beta} \notin J H$, it follows that $\theta \cdot \pi \cdot d_{\beta}<\phi$. So $\theta \cdot \pi \cdot d_{\beta}$ is embeddable in a proper initial segment of $\phi$. Prove by induction that for all $n \in \omega, \theta \cdot \pi \cdot d_{\beta} \cdot n$ is embeddable in a proper initial segment of $\phi$. Then $\theta \cdot \pi \cdot d_{\beta} \cdot \omega=\theta \cdot \pi \cdot d_{\beta+1} \leqq \phi$.

Let $d=\sum_{\alpha<\lambda}^{*} d_{\alpha}$ and $e=\sum_{\alpha<\lambda} d_{\alpha}$. By the embedding lemma, 
either $\theta \cdot \pi \cdot d \leqq \phi$ or $\theta \cdot \pi \cdot e \leqq \phi$. Since $\gamma \leqq \theta \cdot \lambda \leqq \theta \cdot \pi \cdot e$, and $\gamma \leqq \phi$, it follows that $\theta \cdot \pi \cdot d \leqq \phi$. But $\theta \cdot \pi \cdot \lambda^{*} \leqq \theta \cdot \pi \cdot d \leqq \phi$, so the lemma follows.

Lemma 10. For every ordinal $\alpha,\left(\lambda^{\alpha}\right)^{*} \leqq \phi$.

Proof. Prove by induction on the ordinals that $\theta \cdot\left(\lambda^{\alpha}\right)^{*} \leqq \phi$. If $\alpha=0$, then $\theta \cdot\left(\lambda^{\alpha}\right)^{*}=\theta$ which is embeddable in $\phi$ by Lemma 4. If $\alpha=\beta+1$, then $\theta \cdot\left(\lambda^{\alpha}\right)^{*}=\theta \cdot\left(\lambda^{\beta}\right)^{*} \cdot \lambda^{*}$, which is embeddable in $\phi$ by the previous lemma and the induction hypothesis.

So suppose $\alpha$ is a limit ordinal, and for all $\beta<\alpha, \theta \cdot\left(\lambda^{\beta}\right)^{*} \leqq \phi$. Let $I \cong \alpha$ be a cofinal set of ordinals whose type is a regular cardinal $\nu$. Then $\left(\lambda^{\alpha}\right)^{*}$ is the converse well-ordered sum of the regular segmented sequence $\left\langle\left(\lambda^{\beta}\right)^{*}: \beta \in I\right\rangle$. Let $\chi$ be the well-ordered sum of this sequence. By the embeddability lemma, either $\theta \cdot\left(\lambda^{\alpha}\right)^{*} \leqq \phi$ or $\theta \cdot \chi=\theta \cdot \sum_{\beta \in I}\left(\lambda^{\beta}\right)^{*}=\sum_{\beta \in I} \theta \cdot\left(\lambda^{\beta}\right)^{*} \leqq \phi$. The former is the conclusion of the lemma. So suppose the latter holds. If $\nu \geqq \lambda$, then $\gamma \leqq \theta \cdot \lambda \leqq$ $\theta \cdot \nu \leqq \theta \cdot \chi \leqq \phi$, which contradicts the fact that $\gamma \not \phi$. Thus $\nu<\lambda$. So by the previous two lemmas, $\theta \cdot \chi \cdot \lambda^{*} \leqq \phi$. But $\theta \cdot\left(\lambda^{\alpha}\right)^{*} \leqq \theta \cdot \chi \cdot \lambda^{*}$, $\theta \cdot\left(\lambda^{\alpha}\right)^{*} \leqq \phi$, and the lemma follows.

This lemma contradicts the fact that the converse ordinals embeddable in $\phi$ are bounded above by $|\phi|^{+}=|L|^{+}$where $L$ is a representative of $\phi$. Thus the original assumption of the existence of a scattered Hagendorf order type other than the order type of an ordinal is false, and Theorem 1 is proved.

\section{REFERENCES}

1. J. Hagendorf, private communication to F. Galvin.

2. F. Hausdorf, Grudzüge einer Theorie der geordneten Mengen, Math. Ann., 65 (1908), 435-505.

3. J. Larson, On a question of Hagendorf, Amer. Math. Soc. Notices, 23 (1976), A-31.

4. R. Laver, On Fräisse's order type conjecture, Ann. of Math., 93 (1971), 89-111.

5. ——, An order type decomposition theorem, Ann. of Math., 98 (1973), 96-119.

Received July 8, 1977 and in revised form September 23, 1977. The author wishes to thank R. Laver whose conversation and papers [4], [5] inspired the paper.

UNIVERSITY OF FLORIDA GaINESville, FL 32611 



\section{PACIFIC JOURNAL OF MATHEMATICS}

\section{EDITORS}

RICHARD ARENS (Managing Editor)

University of California

Los Angeles, CA 90024

Charles W. Curtis

University of Oregon

Eugene, OR 97403

C. C. Moore

University of California

Berkeley, CA 94720

\section{J. DugundJI}

Department of Mathematics

University of Southern California

Los Angeles, CA 90007

R. FInN and J. Milgram

Stanford University

Stanford, CA 94305

\section{ASSOCIATE EDITORS}
E. F. BECKENBACH
B. H. NeumanN
F. WOLF
K. YoSHIDA

\section{SUPPORTING INSTITUTIONS}

\author{
UNIVERSITY OF BRITISH COLUMBIA \\ CALIFORNIA INSTITUTE OF TECHNOLOGY \\ UNIVERSITY OF CALIFORNIA \\ MONTANA STATE UNIVERSITY \\ UNIVERSITY OF NEVADA, RENO \\ NEW MEXICO STATE UNIVERSITY \\ OREGON STATE UNIVERSITY \\ UNIVERSITY OF OREGON
}

\author{
UNIVERSITY OF SOUTHERN CALIFORNIA \\ STANFORD UNIVERSITY \\ UNIVERSITY OF HAWAII \\ UNIVERSITY OF TOKYO \\ UNIVERSITY OF UTAH \\ WASHINGTON STATE UNIVERSITY \\ UNIVERSITY OF WASHINGTON
}

The Supporting Institutions listed above contribute to the cost of publication of this Journal, but they are not owners or publishers and have no responsibility for its content or policies.

Mathematical papers intended for publication in the Pacific Journal of Mathematics should be in typed form or offset-reproduced, (not dittoed), double spaced with large margins. Please do not use built up fractions in the text of the manuscript. However, you may use them in the displayed equations. Underline Greek letters in red, German in green, and script in blue. The first paragraph or two must be capable of being used separately as a synopsis of the entire paper. Items of the bibliography should not be cited there unless absolutely necessary, in which case they must be identified by author and journal, rather than by item number. Manuscripts, in triplicate, may be sent to any one of the editors. Please classify according to the scheme of Math. Reviews, Index to Vol. 39. All other communications should be addressed to the managing editor, or Elaine Barth, University of California, Los Angeles, California, 90024.

50 reprints to each author are provided free for each article, only if page charges have been substantially paid. Additional copies may be obtained at cost in multiples of 50 .

The Pacific Journal of Mathematics is issued monthly as of January 1966. Regular subscription rate: $\$ 72.00$ a year (6 Vols., 12 issues). Special rate: $\$ 36.00$ a year to individual members of supporting institutions.

Subscriptions, orders for numbers issued in the last three calendar years, and changes of address should be sent to Pacific Journal of Mathematics, 103 Highland Boulevard, Berkeley, California, 94708. Older back numbers obtainable from Kraus Periodicals Co., Route 100, Millwood, NY 10546.

PUBLISHED BY PACIFIC JOURNAL OF MATHEMATICS, A NON-PROFIT CORPORATION

Printed at Kokusai Bunken Insatsusha (International Academic Printing Co., Ltd.). 8-8, 3-chome, Takadanobaba, Shinjuku-ku, Tokyo 160, Japan.

Copyright (C) 1978 by Pacific Journal of Mathematics

Manufactured and first issued in Japan 


\section{Pacific Journal of Mathematics

Vol. 74, No. $2 \quad$ June, 1978

Aharon Atzmon, Spectral synthesis in some spaces of bounded continuous

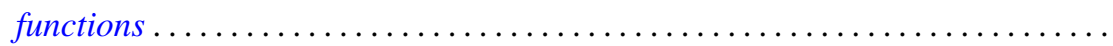

Karl Egil Aubert and Isidor Fleischer, Tensor products of ideal systems and their modules.............................................

Richard F. Basener, Several dimensional properties of the spectrum of a uniform

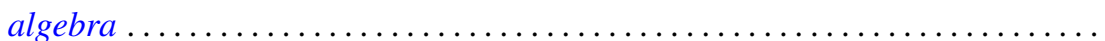

R. H. Bing and Michael Peter Starbird, Super triangulations ............. 307

Andrew Carson, Coherent polynomial rings over regular rings of finite index .....

Robert M. DeVos and Frederick W. Hartmann, Sequences of bounded summability domains .................................................

George Grätzer and R. Padmanabhan, Symmetric difference in abelian groups ....

Robert L. Griess, Jr., A remark about groups of characteristic 2-type and

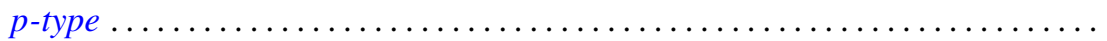

Emil Grosswald and F. J. Schnitzer, A class of modified $\zeta$ and L-functions........

Jutta Hausen and Johnny Albert Johnson, Ideals and radicals of some

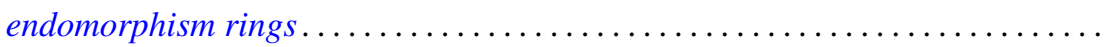

Jean Ann Larson, A solution for scattered order types of a problem of Hagendorf ............................................

Peter A. McCoy, Extremal properties of real biaxially symmetric potentials in $E^{2(\alpha+\beta+2)}$

Héctor Alfredo Merklen, Hereditary crossed product orders .

Hal G. Moore and Adil Mohamed Yaqub, Equational definability of addition in certain rings...

Robert Laurens Moore, Reductivity in $C^{*}$-algebras and essentially reductive operators. . .

Joseph Alvin Neisendorfer, Lie algebras, coalgebras and rational homotopy theory for nilpotent spaces...

William Raymond Nico, Bounded monoids

Richard Paul Osborne, Simplifying spines of 3-manifolds ...

Richard Paul Osborne, The simplest closed 3-manifolds. With an appendix by Osborne and J. Yelle.

Clayton Collier Sherman, The $K$-theory of an equicharacteristic discrete valuation ring injects into the $K$-theory of its field of quotients.... .

Mitchell Herbert Taibleson, The failure of even conjugate characterizations of $H^{1}$

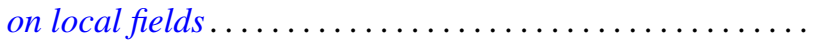

Keti Tenenblat, On characteristic hypersurfaces of submanifolds in Euclidean space ...................................

Jeffrey L. Tollefson, Involutions of Seifert fiber spaces..........

Joel Larry Weiner, An inequality involving the length, curvature, and torsions of a curve in Euclidean $n$-space .......................

Neyamat Zaheer, On generalized polars of the product of abstract homogeneous polynomials.... 